\title{
Usefulness of the Delay Alternating with Nutation for Tailored Excitation Pulse with T1-Weighted Sampling Perfection with Application-Optimized Contrasts Using Different Flip Angle Evolution in the Detection of Cerebral Metastases: Comparison with MPRAGE Imaging
}

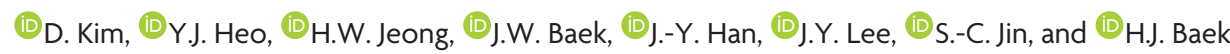

\begin{abstract}
BACKGROUND AND PURPOSE: Contrast-enhanced T1-weighted sampling perfection with application-optimized contrasts by using different flip angle evolution (SPACE) with the delay alternating with nutation for tailored excitation (DANTE) pulse could suppress the blood flow signal and provide a higher contrast-to-noise ratio of enhancing lesion-to-brain parenchyma than the MPRAGE sequence. The purpose of our study was to evaluate the usefulness of SPACE with DANTE compared with MPRAGE for detecting brain metastases.

MATERIALS AND METHODS: Seventy-one patients who underwent contrast-enhanced SPACE with DANTE and MPRAGE sequences and who were suspected of having metastatic lesions were included. Two neuroradiologists determined the number of enhancing lesions, and diagnostic performance was evaluated using figure of merit, sensitivity, positive predictive value, interobserver agreement, and reading time. Contrast-to-noise ratio ${ }_{\text {lesion/parenchyma }}$ and contrast-to-noise ratio ${ }_{\text {white matter/gray matter }}$ were also assessed.

RESULTS: SPACE with DANTE (observer one, 328; observer two, 324) revealed significantly more small ( $<5$ mm) enhancing lesions than MPRAGE (observer one, 175; observer two, 150) $(P<0.001$ for observer 1, $P \leq .0001$ for observer 2). Furthermore, SPACE with DANTE showed significantly higher figure of merit and sensitivity and shorter reading time than MPRAGE for both observers. The mean contrast-to-noise ratio $_{\text {lesion/parenchyma }}$ of SPACE with DANTE $(52.3 \pm 43.1)$ was significantly higher than that of MPRAGE $(17.5 \pm 19.3)(P \leq .0001)$, but the mean contrast-to-noise ratio $_{\text {white matter/gray matter }}$ of SPACE with DANTE $(-0.65 \pm 1.39)$ was significantly lower than that of MPRAGE (3.08 \pm 1.39$)$ $(P \leq .0001)$.
\end{abstract}

CONCLUSIONS: Compared with MPRAGE, SPACE with DANTE significantly improves the detection of brain metastases.

ABBREVIATIONS: $\mathrm{CE}$ = contrast-enhanced; $\mathrm{CNR}=$ contrast-to-noise ratio; $\mathrm{DANTE}=$ delay alternating with nutation for tailored excitation; $\mathrm{FOM}=$ figure of merit; JAFROC = jackknife free-response receiver operating characteristic; SPACE = sampling perfection with application-optimized contrasts by using different flip angle evolution

$T^{2}$ he incidence and prevalence of brain metastases are increasing due to their early detection using advanced imaging techniques and median survival is increasing because of improved therapies. ${ }^{1}$ Accurate diagnosis of brain metastasis before treatment is important because therapeutic planning is dependent on the presence and number of metastatic lesions. Early detection of brain metastases is also important for prognosis because the local control rate is significantly higher for small metastatic lesions

Received February 22, 2019; accepted after revision June 27.

From the Departments of Radiology (D.K., Y.J.H., H.W.J., J.W.B., J.-Y.H.), and Internal Medicine (J.Y.L.), Inje University Busan Paik Hospital, Busan, Korea; Department of Neurosurgery (S.-C.J.), Inje University Haeundae Paik Hospital, Busan, Republic of Korea; and Department of Radiology (H.J.B.), Gyeongsang National University School of Medicine and Gyeongsang National University Changwon Hospital, Changwon, Republic of Korea.

Please address correspondence to Young Jin Heo, MD, Department of Radiology, Inje University Busan Paik Hospital, Bokji-ro 75, Busanjin-Gu, Busan, Republic of Korea, 47392; e-mail: youngjin726@hanmail.net

http://dx.doi.org/10.3174/ajnr.A6158 $\left(<10\right.$-mm diameter) treated using radiosurgery. ${ }^{2,3}$ Contrast-enhanced (CE) 3D T1-weighted scanning shows higher sensitivity than 2D T1-weighted enhanced scanning for the detection of small brain metastases and has been used more frequently for the evaluation of brain metastases. ${ }^{4,5}$

The 3D CE T1-weighted images provide submillimeter resolution and are appropriate for the evaluation of small metastases. However, 3D CE gradient-echo images have a limitation that may mimic the normal enhancing vessels as small metastatic lesions, particularly in regions close to the cortices or sulci. ${ }^{6}$ In contrast to 3D gradient-echo images, 3D CE spin-echo images incorporate blood suppression and make it easy to differentiate blood signals from enhanced tumors, particularly for small lesions. ${ }^{7-9}$ Thus, 3D spin-echo images demonstrate contrast-enhancing lesions more clearly than gradient-echo images, resulting in improved detection. ${ }^{10}$

However, sampling perfection with application-optimized contrasts by using different flip angle evolution (SPACE se- 
quence; Siemens, Erlangen, Germany) alone is not sufficient for evaluation of enhancing lesions because residual blood signals can occasionally be mistaken for these lesions. Thus, black-blood imaging has been used to nullify the signal of blood flow in the evaluation of brain metastases. A few studies, ${ }^{9,11,12}$ using blackblood imaging have shown improved diagnostic performance for the detection of brain metastases due to a higher contrast-to-noise ratio (CNR). A 3D black-blood imaging method, delay alternating with nutation for tailored excitation (DANTE), is an advanced technique. It can suppress the residual blood flow, which is incompletely suppressed by SPACE. ${ }^{13}$ No previous study has used the DANTE preparation pulse in the evaluation of brain metastasis, to our knowledge. We hypothesized that the combination of DANTE preparation and the inherent black-blood effect due to SPACE may be advantageous for the detection of contrast-enhancing lesions in the brain parenchyma. Thus, the purpose of our study was to evaluate the usefulness of SPACE with DANTE in the detection of brain metastases compared with MPRAGE imaging.

\section{MATERIALS AND METHODS Patient Population}

This study was approved by the institutional review board of Inje University Busan Paik Hospital, and the need for informed consent was waived. Between May 2018 and January 2019, three hundred fifty-eight patients with pathologically-proved primary cancer underwent 3D CE T1-weighted MR imaging using the SPACE with DANTE and MPRAGE sequences in the same imaging session for evaluation of brain metastases. The diagnoses of the brain metastases were based on previous and follow-up MR imaging findings determined in consensus by 2 experienced neuroradiologists, one with 19 years and one with 4 years of experience in neuroimaging, who did not participate in image analysis. Brain metastases were determined according to the following criteria: 1 ) newly occurring enhancing lesions or an increase in size of the existing lesions at follow-up MR imaging or a decrease in size or disappearance after treatment; 2) what appeared as a lesion not being a normal enhancing structure or an artifact; and 3) contrast-enhancing lesions located in the brain parenchyma. Contrast-enhancing lesions that were visualized on only 1 sequence were also evaluated. We considered these as false-positive lesions, and the possible causes were recorded. Patients who exhibited no enhancing lesions $(n=200)$, those who exhibited enhancing lesions but did not undergo follow-up MR imaging $(n=58)$, and those who had leptomeningeal metastasis $(n=$ $29)$ were excluded. Finally, 71 patients with a mean age of 64.3 years (age range, 40-84 years; 46 men and 25 women) were included. The diagnoses of primary tumors were lung cancer $(n=53)$, breast cancer $(n=8)$, colon cancer $(n=2)$, prostate cancer $(n=2)$, renal cell cancer $(n=1)$, gastric cancer $(n=1)$, ovarian cancer $(n=1)$, endometrial cancer $(n=1)$, uterine cervix cancer $(n=1)$, and external auditory canal tumor $(n=$ 1); 540 enhancing lesions were designated as brain metastases, and 28 enhancing lesions were detected on either of the two 3D-enhanced sequences. The median interval between the initial and follow-up MR imaging was 115.0 days (range, 26 2061 days).

\section{MR Imaging Protocol}

All studies were performed using a 3T MR imaging scanner (Magnetom Skyra; Siemens, Erlangen, Germany) and a 64-channel head coil. After we obtained routine precontrast images using axial fluid-attenuated inversion recovery, T2-weighted, T1weighted, gradient-echo, 3D SPACE, and 3D MPRAGE images with fat suppression were obtained immediately following intravenous administration of $0.1 \mathrm{mmol} / \mathrm{kg}$ body weight of Dotarem (gadoterate meglumine; Guerbet, Aulnay-sous-Bois, France). The scan parameters of postcontrast MR imaging were as follows for SPACE with DANTE imaging: TR, $800 \mathrm{~ms}$; TE, $15 \mathrm{~ms}$; flip angle, variable; fat suppression, Fat-Sat (chemical shift selective suppression); parallel acquisition techniques factor, 2; FOV, $230 \times 230$; bandwidth, $422 \mathrm{~Hz} /$ pixel; matrix, $320 \times 320$; number of slices, 240; echo spacing, $4.88 \mathrm{~ms}$; voxel size, $0.72 \times$ $0.72 \times 0.72 \mathrm{~mm}$; scan time, 5 minutes 54 seconds; DANTE preparation pulse were applied; for MPRAGE imaging: TR, $2200 \mathrm{~ms}$; TE, $3.05 \mathrm{~ms}$; flip angle, $9^{\circ}$; fat suppression, water excitation; parallel acquisition techniques factor, 2; FOV, $230 \times 230$; bandwidth, $260 \mathrm{~Hz} /$ pixel; matrix, $320 \times 320$; number of slices, 240 ; echo spacing, $8.8 \mathrm{~ms}$; voxel size, $0.72 \times 0.72 \times 0.72 \mathrm{~mm}$; flip angle, $9^{\circ}$; scan time, 5 minutes 32 seconds. We obtained sagittal planes covering the whole brain for the SPACE and MPRAGE imaging to reduce the scan time. We performed SPACE with DANTE and MPRAGE sequences in alternative order by random distribution to avoid timing bias, which can increase contrast agent uptake due to the delay after injection. The order of sequences was the following: SPACE with DANTE followed by MPRAGE in 32 patients and MPRAGE followed by SPACE with DANTE in 39 patients.

\section{Image Analysis}

Determination of Metastatic Lesions. Two neuroradiologists, one with 5 years of experience and one with 1 year of experience in neuroimaging, independently evaluated the presence of brain metastases using SPACE with DANTE and MPRAGE imaging with a 4-week interval to minimize any learning bias. One observer evaluated SPACE with DANTE followed by MPRAGE, and another observer evaluated MPRAGE followed by SPACE with DANTE. Both observers reported all enhancing lesions in the brain parenchyma, except for the normal anatomic structures or artifacts. The metastatic lesions were classified into 2 groups by lesion size: large ( $\geq 5 \mathrm{~mm}$ ) and small ( $\leq 5 \mathrm{~mm}$ ). Both observers also reported the level of confidence of metastatic lesions at each location on a rating scale (ranging from a lowest confidence level of 0 to a highest confidence level of 100), and the reading time of each case was recorded.

Evaluation of Image Quality. We evaluated and compared the CNR of lesions with normal parenchyma $\left(\mathrm{CNR}_{\text {lesion/parenchyma }}\right)$ and the $\mathrm{CNR}$ of white matter with gray matter $\left(\mathrm{CNR}_{\text {white matter/gray matter }}\right)$ among the SPACE with DANTE and MPRAGE images. For evalua-

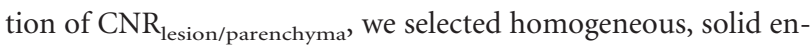
hancing lesions of $>5 \mathrm{~mm}$ and excluded rimlike enhancing lesions due to the difficulty in drawing the ROI. The CNR of enhancing lesions was calculated according to Kammer et $\mathrm{al}^{6}$ :

$$
\mathrm{CNR}_{\text {lesion/parenchyma }}=\left(\mathrm{SI}_{\text {lesion }}-\mathrm{SI}_{\text {parenchyma }}\right) / \mathrm{SD}_{\text {parenchyma }} \text {. }
$$



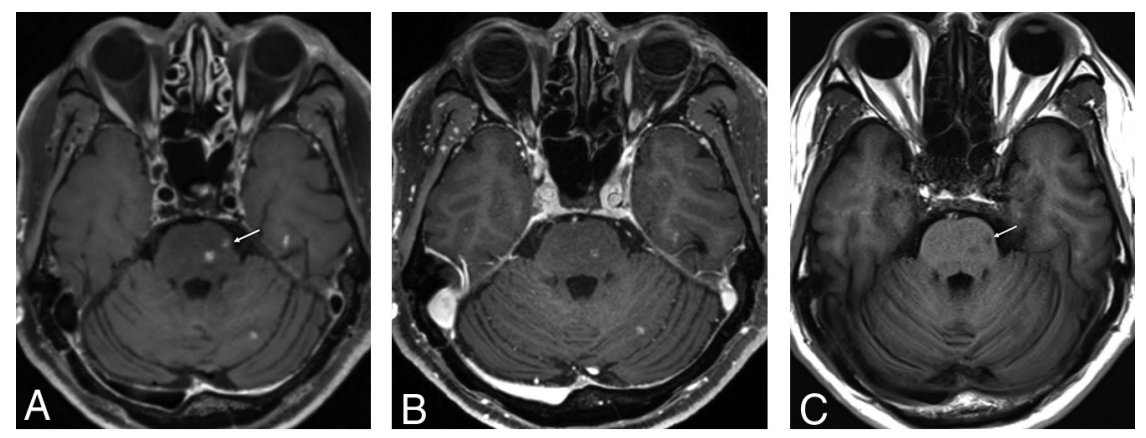

$22.73 \mathrm{~mm}^{2}$. Every ROI of enhancing lesions was placed at the center of the lesion, to the extent possible, by 1 neuroradiologist (one with 5 years of experience), and the area of the ROI was dependent on lesion size, varying between 3.72 and $22.73 \mathrm{~mm}^{2}$.

\section{Statistical Analysis}

All statistical analyses were performed using SAS, Version 9.4 (SAS Institute, Cary, North Carolina). The variables are presented as number or mean $\pm \mathrm{SD}$. For evaluation of the diagnostic performance of each observer in each reading session, we used figure of merit (FOM) derived from the jackknife free-response receiver operating characteristic (JAFROC) analysis with method 1 of Chakraborty and Berbaum. ${ }^{11,12,15,16}$ A free software JAFROC analysis package is available at http://www.devchakraborty.com. The paired $t$ test was used to compare the number of lesions, CNR, and reading time between SPACE with DANTE and MPRAGE. The sensitivity and positive predictive value of different MR images were calculated using a 2-way contingency table. $P$ values $<.05$ were considered statistically significant. Interobserver agreement for each MR image was calculated using $\kappa$ statistics; $0-0.20,0.21-0.40$, $0.41-0.60,0.61-0.80$, and $0.81-1.00$ were regarded as slight, fair, moderate, substantial, and almost perfect agreement, respectively, based on the Landis and Koch method. ${ }^{17}$

FIG 2. MR images of a 54-year-old male patient with lung cancer. Contrast-enhanced SPACE DANTE $(A)$ clearly shows two enhancing lesions in the right parietal lobe (arrow and arrowhead). One enhancing lesion is clearly visible (arrow), but the other enhancing lesion at the posterior aspect (arrowhead) is poorly visible on MPRAGE (B). However, this lesion has increased (arrowhead) in size and shows increased enhancement on the follow-up MR imaging 3 months later (C).

\section{RESULTS}

\section{Diagnostic Performance of the MR Images}

We calculated the CNR for differentiating the gray and white matter as follows:

$$
\mathrm{CNR}_{\text {white matter/gray matter }}=\left(\mathrm{SI}_{\text {white matter }}-\mathrm{SI}_{\text {gray matter }}\right) / \mathrm{SD}_{\text {white matter }} \text {. }
$$

SPACE with DANTE (observer one, 328; observer two, 324) revealed significantly more small $(<5 \mathrm{~mm})$ enhancing lesions in the brain parenchyma than MPRAGE (observer one, 175; observer two, 150) $(P=.0006$ for observer $1, P \leq .0001$ for observer 2$)$ (Figs 1 and 2 and Table 1). In the detection of lesions of $>5 \mathrm{~mm}$, SPACE with DANTE (observer one, 186; observer two, 188) also revealed more enhancing lesions than MPRAGE (observer one, 168; observer two, 169), but this difference failed to reach statistical significance. Moreover, SPACE with DANTE showed higher sensitivity than MPRAGE for the detection of brain metastases, regardless of the lesion size (Fig 3). Both SPACE with DANTE and MPRAGE showed almost perfect interobserver agreement $(\kappa=$ 0.99 for SPACE with DANTE and 0.98 for MPRAGE for lesions smaller than $5 \mathrm{~mm}$; $\kappa=0.99$ for SPACE with DANTE and 0.98 for MPRAGE for lesions of $>5 \mathrm{~mm}$ ) for the detection of brain metastases, regardless of lesion size. The FOM of SPACE with 
Table 1: Comparison of lesion detectability according to lesion size

\begin{tabular}{|c|c|c|c|}
\hline & SPACE with DANTE & MPRAGE & $P$ Value \\
\hline \multicolumn{4}{|c|}{ Lesion diameter $\leq 5 \mathrm{~mm}$} \\
\hline \multicolumn{4}{|l|}{ Observer 1} \\
\hline No. of lesions ${ }^{a}$ & $328(4.39 \pm 7.54)$ & $175(2.96 \pm 5.13)$ & .0006 \\
\hline Sensitivity (\%) & 87.4 & 49.4 & \\
\hline PPV $(\%)^{b}$ & 98.1 & 95.6 & \\
\hline \multicolumn{4}{|l|}{ Observer 2} \\
\hline No. of lesions ${ }^{a}$ & $324(4.50 \pm 7.52)$ & $150(2.76 \pm 5.20)$ & $<.0001$ \\
\hline Sensitivity (\%) ${ }^{\mathrm{b}}$ & 86.86 & 51.14 & \\
\hline PPV $(\%)^{b}$ & 97.8 & 98.4 & \\
\hline $\mathrm{ICC}^{\mathrm{C}}$ & 0.99 & 0.98 & \\
\hline \multicolumn{4}{|c|}{ Lesion diameter $>5 \mathrm{~mm}$} \\
\hline \multicolumn{4}{|c|}{ Observer 1} \\
\hline No. of lesions ${ }^{a}$ & $186(2.62 \pm 4.51)$ & $168(2.37 \pm 3.52)$ & .0978 \\
\hline Sensitivity (\%) ${ }^{\mathrm{b}}$ & 94.74 & 85.79 & \\
\hline PPV $(\%)^{b}$ & 96.77 & 97.02 & \\
\hline \multicolumn{4}{|l|}{ Observer 2} \\
\hline No. of lesions ${ }^{a}$ & $188(2.65 \pm 4.37)$ & $169(2.38 \pm 3.92)$ & .0531 \\
\hline Sensitivity $(\%)^{\mathrm{b}}$ & 95.26 & 87.37 & \\
\hline PPV $(\%)^{b}$ & 96.28 & 98.22 & \\
\hline$I C C^{\mathrm{C}}$ & 0.99 & 0.98 & \\
\hline
\end{tabular}

Note:-ICC indicates intraclass correlation coefficient; PPV, positive predictive value.

a Data represent the number (mean). Values were compared using paired $t$ tests.

b Values were calculated using a 2-way contingency table.

c Values were calculated using $\kappa$ statistics.
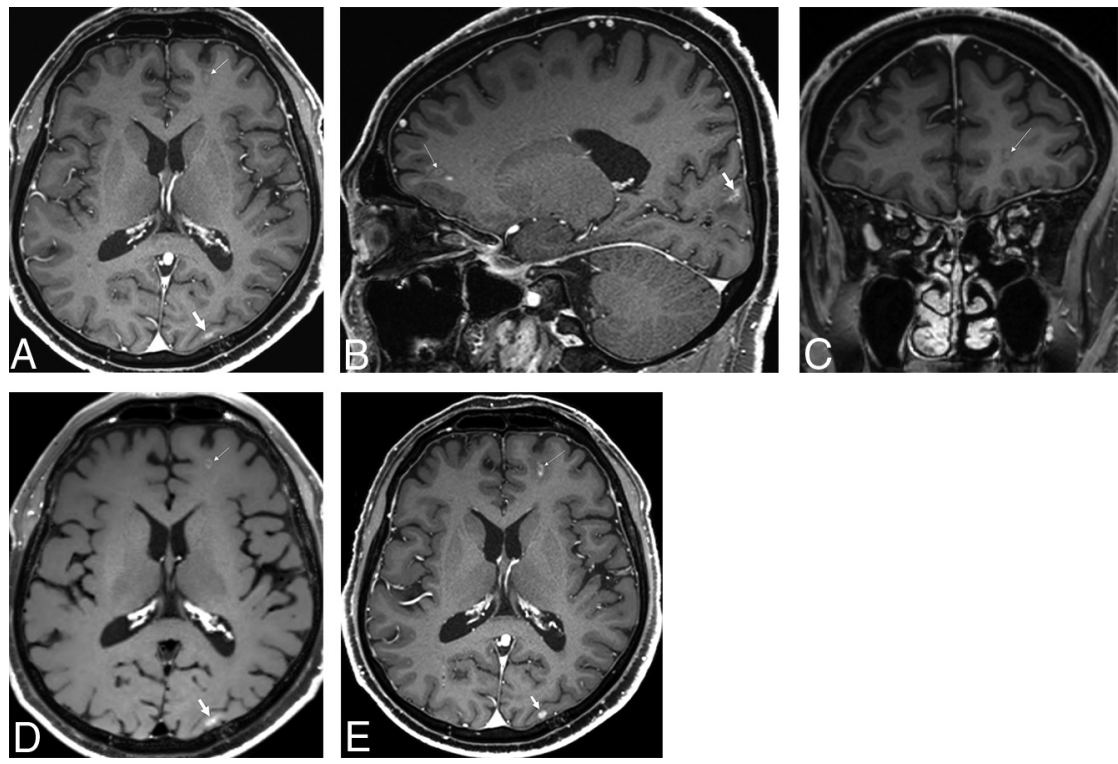

FIG 3. Minute and faint enhancing lesions at the left frontal and occipital lobes were missed by observer $2(A)$. A left frontal lesion also shows faint enhancement on sagittal and coronal reconstructed MPRAGE images ( $B$ and $C$ ). However, these enhancing lesions show more prominent enhancement on SPACE with DANTE $(D)$ and increased size on the follow-up MR imaging $(E) 4$ months later.

Table 2: Comparison of FOM according to lesion size ${ }^{a}$

\begin{tabular}{cccc}
\hline & $\begin{array}{c}\text { SPACE with } \\
\text { DANTE }\end{array}$ & MPRAGE & $\boldsymbol{P}$ Value \\
\hline $\begin{array}{c}\text { Lesion diameter } \leq 5 \mathrm{~mm} \\
\text { Observer 1 }\end{array}$ & 0.904 & 0.698 & \\
Observer 2 & 0.861 & 0.702 & \\
Mean \pm SD & $0.882 \pm 0.023$ & $0.700 \pm 0.038$ & .0017 \\
Lesion diameter $>5 \mathrm{~mm}$ & & & \\
Observer 1 & 0.957 & 0.921 & \\
Observer 2 & 0.943 & 0.928 & \\
Mean \pm SD & $0.950 \pm 0.013$ & $0.925 \pm 0.014$ & .1762 \\
\hline
\end{tabular}

${ }^{a}$ Data for observers 1 and 2 are mean value of FOM data compared between SPACE with DANTE and MPRAGE.
DANTE was significantly higher than that of MPRAGE for both observers in the detection of lesions smaller than 5 $\mathrm{mm}(P=.0017)$ (Table 2). However, the FOM was not significantly different between the 2 sequences in the detection of lesions of $>5 \mathrm{~mm}(P=.1762)$.

SPACE with DANTE revealed more false-positive findings $(n=18)$ due to incomplete vessel suppression $(n=15)$ (Fig 4) and flow-related artifacts $(n=3)$. On MPRAGE $(n=10)$, the causes for the false-positive findings were vascular structures $(n=9)$ and flow-related artifacts $(n=1)$. However, these findings were not significantly different between the two 3D-enhanced MR images. The average reading time of SPACE with DANTE (observer one, $45.4 \pm 31.7 \mathrm{sec}$ onds; observer two, $53.7 \pm 21.5$ seconds) was significantly shorter than that of MPRAGE for both observers (observer one, $73.0 \pm 54.1$ seconds; observer two, $72.0 \pm 22.8$ seconds $)(P \leq$ .0001).

\section{Evaluation of Image Quality}

A total of 51 patients who exhibited homogeneous, solid enhancing lesions of $>5 \mathrm{~mm}$ were evaluated. The mean $\mathrm{CNR}_{\text {lesion/parenchyma }}$ of SPACE with DANTE $(52.3 \pm 43.1)$ was significantly higher than that of MPRAGE $(17.5 \pm 19.3)$ $(P \leq .0001)$ (Table 3 and Fig 5). However, the mean $\mathrm{CNR}_{\text {white matter/gray matter of }}$ SPACE with DANTE $(-0.65 \pm 1.39)$ was significantly lower than that of MPRAGE $(3.08 \pm 1.39)(P \leq .0001)$.

\section{DISCUSSION}

We compared the diagnostic performance of CE 3D-SPACE with DANTE and MPRAGE for detecting brain metastases. The SPACE with DANTE sequences showed significantly higher sensitivity than the MPRAGE sequences, especially for smaller lesions $(<5 \mathrm{~mm})$, and they also showed higher interobserver agreement than the MPRAGE sequences. The SPACE with DANTE sequences showed improved arterial and venous blood suppression compared with SPACE alone. ${ }^{7,13}$ Moreover, DANTE can supplement this suppression using the gradient pulse in the phase-encoding direction. ${ }^{13,18}$ It also suppresses the signal due to slow blood flow, which is incompletely suppressed by SPACE. ${ }^{13,19}$ In addition, SPACE generates intravoxel dephasing and helps maintain the black-blood effect that DANTE cannot sustain during the readout period. ${ }^{13}$ These factors may simplify the reading process; thus, the reading time of SPACE 

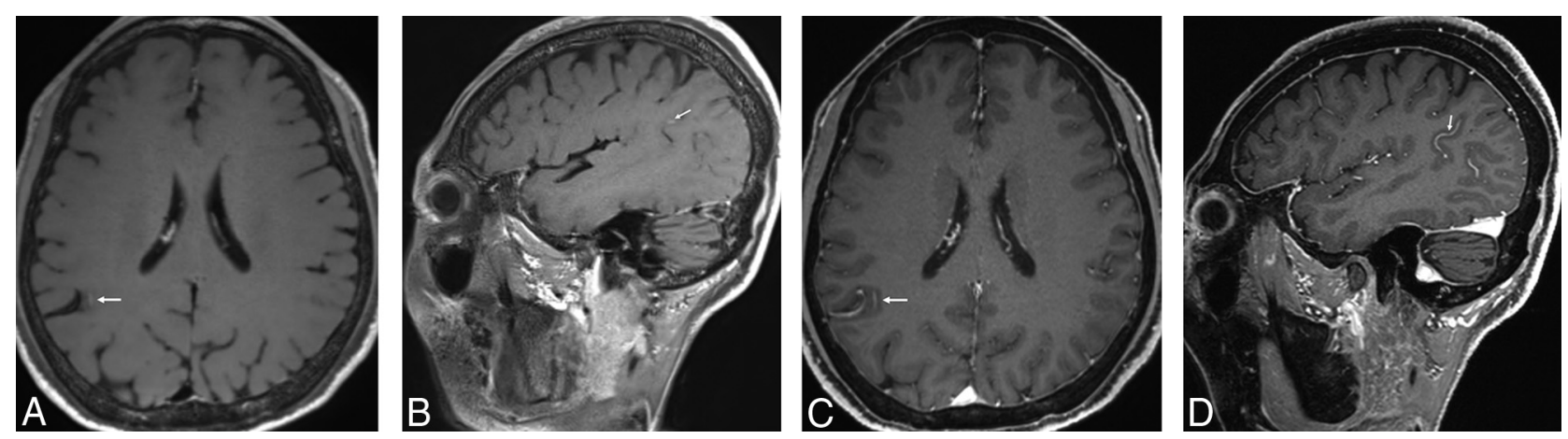

FIG 4. Contrast-enhanced SPACE with DANTE $(A$ and $B)$ shows a focal, linear enhancing lesion in the right parietal lobe. However, we could easily recognize this as a false-positive finding caused by incomplete vessel suppression on MPRAGE images ( $C$ and $D)$.

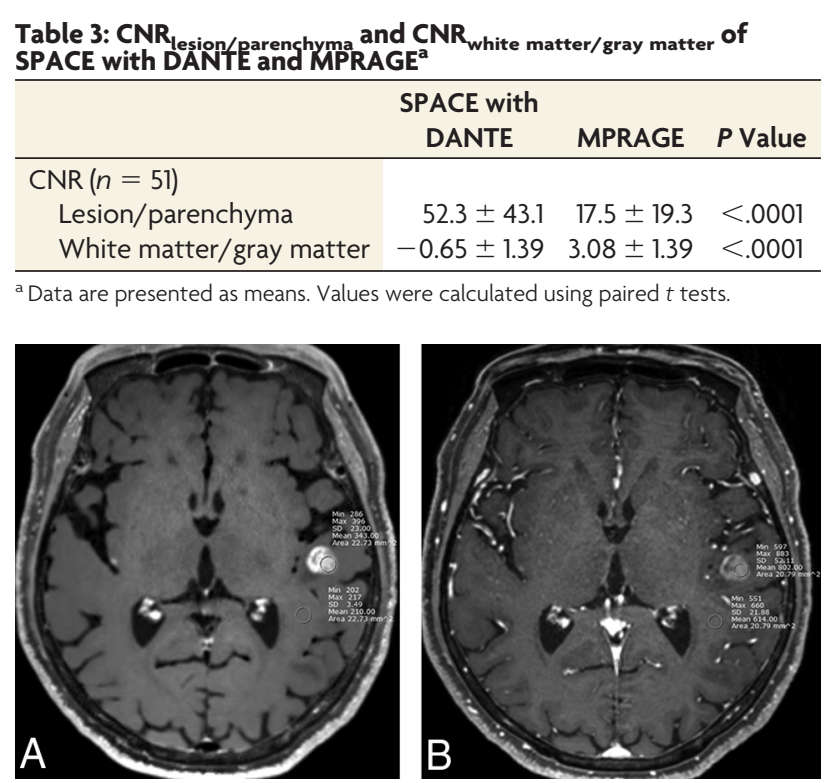

FIG 5. The $\mathrm{CNR}_{\text {lesion/parenchyma }}$ of SPACE with DANTE (8.62) (A) was higher than that of MPRAGE (3.49) (B).

with DANTE was significantly shorter than that of MPRAGE for both observers.

We measured the CNR of enhancing lesions for both SPACE with DANTE and MPRAGE images using the following formula: $\mathrm{CNR}_{\text {lesion/parenchyma }}=\left(\mathrm{SI}_{\text {lesion }}-\mathrm{SI}_{\text {parenchyma }}\right) / \mathrm{SD}_{\text {parenchyma. }}$ Our study showed that the $\mathrm{CNR}_{\text {lesion/parenchyma }}$ of SPACE with DANTE is higher than that of MPRAGE. This finding is in agreement with those of previous studies, ${ }^{6,11,20}$ which showed lower contrast enhancement of gradient-echo images than of spin-echo images and higher $\mathrm{CNR}_{\text {lesion/parenchyma }}$ of SPACE images than of MPRAGE images. Previous studies ${ }^{4,8,10}$ have provided several reasons for the better detection of contrast-enhancing lesions using the spin-echo sequence than using the gradient-echo sequence. Previous studies ${ }^{10,20,21}$ suggested that the higher CNR of enhancing metastatic lesions to the normal parenchyma of the spin-echo sequence than of the gradient-echo sequence contributes to the higher detectability of metastatic lesions. Our result that the CNR of SPACE with DANTE is higher than that of MPRAGE is consistent with the findings of previous studies. ${ }^{10,20,21}$ The higher magnetization transfer effect of SPACE than MPRAGE has been suggested as another factor influenc- ing its higher detection accuracy. ${ }^{8,22} \mathrm{~A}$ variable flip angle, which might be introduced as off-resonance pulses, induces magnetization transfer effects. It preferentially reduces the signal from the brain parenchyma, especially from white matter, rendering enhancing lesions more outstanding. However, SPACE alone is not sufficient for the evaluation of enhancing lesions because residual blood signal can occasionally be mistaken for enhancing lesions.

Therefore, black-blood modules have been used for evaluation of brain metastases. A few studies ${ }^{9}$ have evaluated the detectability of brain metastasis using 3D CE MR imaging with the black-blood module, but no study has used the DANTE preparation pulse in the evaluation of brain metastasis. A previous study ${ }^{18}$ suggested that DANTE preparation is a promising black-blood module that offers a higher signal-to-noise ratio and allows a shorter acquisition time than other types of black-blood modules, such as double inversion recovery or motion-sensitive driven equilibrium preparation modules. Our findings are in agreement with those of Park et al, ${ }^{9}$ who detected significantly more small lesions using the CE 3D black-blood single slab turbo spin-echo sequence than using the CE MPRAGE sequence. They found no significant differences in the detection of larger enhancing lesions $(>5 \mathrm{~mm})$, and this finding is in agreement with that of our study.

We could recognize the enhancing lesions more easily using SPACE with DANTE than by using MPRAGE, and the result of our study that SPACE with DANTE required a shorter reading time than MPRAGE for both observers supports this finding. This finding is consistent with those of previous studies that analyzed the reading time. ${ }^{11,12}$ The FOM of SPACE with DANTE was significantly higher than that of MPRAGE for both observers in the detection of lesions of $<5 \mathrm{~mm}$ in our study. A previous study ${ }^{8}$ reported that SPACE images showed significantly higher diagnostic performance than MPRAGE images, regardless of the observer's experience in neuroradiology. However, Kakeda et $\mathrm{al}^{4}$ reported no evident difference in the conspicuity of the enhancing lesions between spin-echo and gradient-echo images in a lesionby-lesion comparison. This is probably because 3D gradient-echo images and 2D spin-echo images were compared, unlike in our study.

SPACE with DANTE revealed more false-positive lesions than MPRAGE, and $83.3 \%$ of these were caused by incomplete

AJNR Am J Neuroradiol 40:1469-75 Sep 2019 www.ajnr.org

1473 
suppression of the blood flow. This finding is consistent with a previous study ${ }^{23}$ reported that CE-SPACE showed more falsepositive findings than MPRAGE. The variable flip angle of the SPACE sequence imperfectly suppresses the vascular signal and could lead to misinterpretation of the remaining vessels as a metastatic lesion. Furthermore, turbo spin-echo motion-sensitive driven equilibrium ${ }^{11}$ shows more false-positive lesions than the non-motion-sensitive driven equilibrium sequence due to incomplete blood flow suppression of small peripheral vessels, and these findings hamper interpretation. However, we could easily recognize these structures as false-positive lesions by considering multiplanar reconstruction and MPRAGE images. This method has already been confirmed by a previous study, ${ }^{11}$ which decreased the false-positive rate and preserved diagnostic performance. Kato et $\mathrm{al}^{8}$ also reported several false-positive events, but the causes were different from those identified in our study. None of the venous sinuses or choroid plexuses were misdiagnosed as metastasis in our study.

Our study has several limitations. First, this study was retrospective in design. Second, pathologic confirmation of all metastatic lesions was not possible because patients with multiple brain metastases usually do not undergo an operation. Third, we could not include SPACE without a DANTE pulse due to its limited acquisition time. However, a previous study ${ }^{13}$ has already shown the improved suppression of arterial and venous blood using SPACE with DANTE compared with SPACE. Another study ${ }^{11}$ using turbo spin-echo motion-sensitive driven equilibrium showed that it achieves better blood vessel suppression than non-turbo spin-echo motion-sensitive driven equilibrium, with a similar CNR. Nevertheless, further studies comparing SPACE without DANTE and SPACE with DANTE are needed and may support our results. Finally, in the present study, the observers were not blinded to the type of MR images because the differentiation of gray and white matter was evidently different between the 2 sequences.

\section{CONCLUSIONS}

Using SPACE with DANTE could improve the diagnostic performance for brain metastases; this approach also has almost perfect interobserver agreement. Compared with MPRAGE, SPACE with DANTE significantly improves the detection of brain metastases, particularly of those of $<5 \mathrm{~mm}$, without significantly increasing the false-positive rate. This information should be considered in the development of optimal brain tumor imaging protocols.

\section{ACKNOWLEDGMENTS}

The authors thank In Seong Kim and Kang Soo Kim (Siemens, South Korea) for the DANTE application optimization.

\section{REFERENCES}

1. Langer CJ, Mehta MP. Current management of brain metastases, with a focus on systemic options. J Clin Oncol 2005;23:6207-19 CrossRef Medline

2. Chang EL, Hassenbusch SJ 3rd, Shiu AS, et al. The role of tumor size in the radiosurgical management of patients with ambiguous brain metastases. Neurosurgery 2003;53:272-80; discussion 280-81 CrossRef Medline
3. Minniti G, Clarke E, Lanzetta G, et al. Stereotactic radiosurgery for brain metastases: analysis of outcome and risk of brain radionecrosis. Radiat Oncol 2011;6:48 CrossRef Medline

4. Kakeda S, Korogi Y, Hiai Y, et al. Detection of brain metastasis at 3T: comparison among SE, IR-FSE and 3D-GRE sequences. Eur Radiol 2007;17:2345-51 CrossRef Medline

5. Ellingson BM, Bendszus M, Boxerman J, et al; Jumpstarting Brain Tumor Drug Development Coalition Imaging Standardization Steering Committee. Consensus recommendations for a standardized brain tumor imaging protocol in clinical trials. Neuro Oncol 2015; 17:1188-98 CrossRef Medline

6. Kammer N, Coppenrath E, Treitl K, et al. Comparison of contrastenhanced modified T1-weighted 3D TSE black-blood and 3D MPRAGE sequences for the detection of cerebral metastases and brain tumours. Eur Radiol 2016;26:1818-25 CrossRef Medline

7. Suh CH, Jung SC, Kim KW, et al. The detectability of brain metastases using contrast-enhanced spin-echo or gradient-echo images: a systematic review and meta-analysis. J Neurooncol 2016;129:363-71 CrossRef Medline

8. Kato Y, Higano S, Tamura H, et al. Usefulness of contrast-enhanced T1-weighted sampling perfection with application-optimized contrasts by using different flip angle evolutions in detection of small brain metastasis at 3T MR imaging: comparison with magnetization-prepared rapid acquisition of gradient echo imaging. AJNR Am J Neuroradiol 2009;30:923-29 CrossRef Medline

9. Park J, Kim J, Yoo E, et al. Detection of small metastatic brain tumors: comparison of 3D contrast-enhanced whole-brain blackblood imaging and MP-RAGE imaging. Invest Radiol 2012;47: 136-41 CrossRef Medline

10. Mugler JP 3rd, Brookeman JR. Theoretical analysis of gadopentetate dimeglumine enhancement in T1-weighted imaging of the brain: comparison of two-dimensional spin-echo and three-dimensional gradient-echo sequences. J Magn Reson Imaging 1993;3:761-69 CrossRef Medline

11. Nagao E, Yoshiura T, Hiwatashi A, et al. 3D turbo spin-echo sequence with motion-sensitized driven-equilibrium preparation for detection of brain metastases on 3T MR imaging. AJNR Am J Neuroradiol Neuroradiology 2011;32:664-70 CrossRef Medline

12. Kikuchi K, Hiwatashi A, Togao O, et al. 3D MR sequence capable of simultaneous image acquisitions with and without blood vessel suppression: utility in diagnosing brain metastases. Eur Radiol 2015;25:901-10 CrossRef Medline

13. Xie Y, Yang Q, Xie G, et al. Improved black-blood imaging using DANTE-SPACE for simultaneous carotid and intracranial vessel wall evaluation. Magn Reson Med 2016;75:2286-94 CrossRef Medline

14. Rand S, Maravilla KR, Schmiedl U. Lesion enhancement in radiofrequency spoiled gradient-echo imaging: theory, experimental evaluation, and clinical implications. AJNR Am J Neuroradiol 1994; 15:27-35 Medline

15. Chakraborty DP, Berbaum KS. Observer studies involving detection and localization: modeling, analysis, and validation. Med Phys 2004; 31:2313-30 CrossRef Medline

16. Chakraborty DP. Analysis of location specific observer performance data: validated extensions of the jackknife free-response (JAFROC) method. Acad Radiol 2006;13:1187-93 CrossRef Medline

17. Landis JR, Koch GG. The measurement of observer agreement for categorical data. Biometrics 1977;33:159-74 Medline

18. Li L, Chai JT, Biasiolli L, et al. Black-blood multicontrast imaging of carotid arteries with DANTE-prepared 2D and 3D MR imaging. $R a$ diology 2014;273:560-69 CrossRef Medline

19. Li L, Miller KL, Jezzard P. DANTE-prepared pulse trains: a novel approach to motion-sensitized and motion-suppressed quantitative magnetic resonance imaging. Magn Reson Med 2012;68:1423-38 CrossRef Medline

20. Chappell PM, Pelc NJ, Foo T, et al. Comparison of lesion enhancement on spin-echo and gradient-echo images. AJNR Am J Neuroradiol 1994;15:37-44 Medline 
21. Komada T, Naganawa S, Ogawa H, et al. Contrast-enhanced MR imaging of metastatic brain tumor at 3 Tesla: utility of T(1)weighted SPACE compared with 2D spin echo and 3D gradient echo sequence. Magn Reson Med Sci 2008;7:13-21 CrossRef Medline

22. Constable R, Anderson A, Zhong J, et al. Factors influencing contrast in fast spin-echo MR imaging. Magn Reson Imaging 1992;10:497-511 CrossRef Medline

23. Kwak HS, Hwang S, Chung GH, et al. Detection of small brain metastases at $3 \mathrm{~T}$ : comparing the diagnostic performances of contrastenhanced T1-weighted SPACE, MPRAGE, and 2D FLASH imaging. Clin Imaging 2015;39:571-75 CrossRef Medline 